Rational Expectations Equilibrium: Generic Existence and the Information Revealed by Prices Author(s): Roy Radner

Source: Econometrica, Vol. 47, No. 3 (May, 1979), pp. 655-678

Published by: The Econometric Society

Stable URL: http://www.jstor.org/stable/1910413

Accessed: 07/12/2010 12:59

Your use of the JSTOR archive indicates your acceptance of JSTOR's Terms and Conditions of Use, available at http://www.jstor.org/page/info/about/policies/terms.jsp. JSTOR's Terms and Conditions of Use provides, in part, that unless you have obtained prior permission, you may not download an entire issue of a journal or multiple copies of articles, and you may use content in the JSTOR archive only for your personal, non-commercial use.

Please contact the publisher regarding any further use of this work. Publisher contact information may be obtained at http://www.jstor.org/action/showPublisher?publisherCode=econosoc.

Each copy of any part of a JSTOR transmission must contain the same copyright notice that appears on the screen or printed page of such transmission.

JSTOR is a not-for-profit service that helps scholars, researchers, and students discover, use, and build upon a wide range of content in a trusted digital archive. We use information technology and tools to increase productivity and facilitate new forms of scholarship. For more information about JSTOR, please contact support@jstor.org. 


\title{
RATIONAL EXPECTATIONS EQUILIBRIUM: GENERIC EXISTENCE AND THE INFORMATION REVEALED BY PRICES
}

\author{
By ROY RADNER
}

\begin{abstract}
When traders come to a market with different information about the items to be traded, the resulting market prices may reveal to some traders information originally available only to others. The possibility for such inferences rests upon traders having "models" or "expectations" of how equilibrium prices are related to initial information. This relationship is endogenous, which motivates the term "rational expectations equilibrium." This paper shows that, in a particular model of asset trading, if the number of alternative states of initial information is finite then, generically, rational expectations equilibria exist that reveal to all traders all of their initial information.
\end{abstract}

\section{INTRODUCTION ${ }^{1}$}

WHEN TRADERS COME to a market with different information about the items to be traded, the resulting market prices may reveal to some traders something about the information available to other traders. This phenomenon might be important in the case of assets whose eventual values or utilities are not perfectly known to all traders at the time of purchase, as in the trading of land with uncertain quantities of mineral deposits, or in the trading of common stocks. A thorough theoretical analysis of this situation probably requires a more detailed specification of the trading mechanism than is usual in general equilibrium analysis. Nevertheless, it is tempting to try to obtain results that are as independent as possible of the specifics of the trading mechanism, by using some suitable concept of equilibrium.

The possibility for one trader to make inferences from market prices about the information possessed by other traders rests upon his having a "model" or "expectations" of how equilibrium prices are determined, i.e., how equilibrium prices are related to the information initially possessed by the various traders. But this relationship is endogenous to the market system, and if traders have any opportunity to compare the results of the operation of the market with their own models, then a suitable equilibrium concept would require that their models not be obviously controverted by their observations of the market. This motivates the term "rational expectations equilibrium."

The particular rational expectations equilibrium that one would obtain depends upon the traders' models or expectations of the relationship between traders'

\footnotetext{
${ }^{1}$ I am grateful to Jerry Green, Leonid Hurwicz, James Jordan, and David Kreps for very helpful discussions of the problems treated in this paper. The referees made important suggestions for improving the exposition.

The research and preparation of this paper were partly supported by National Science Foundation Grant SOC73-05652A01 to the University of California, Berkeley, administered by the Center for Research in Management Science. A previous version of this paper was presented at the NBERCEME Seminar on Decentralization, Harvard University, Cambridge, Massachusetts, April 29-May $1,1977$.
} 
initial information and equilibrium prices. In addition, there is the possibility that such an equilibrium might not even exist. In particular, one can give examples in which, if the trader must have perfect knowledge of the relationship between initial information and equilibrium prices, then a rational expectations equilibrium need not exist, even with "standard" assumptions about preferences, endowments, etc. ${ }^{2}$ However, in this paper I shall show that, if the number of alternative states of initial information is finite, then the nonexistence of equilibrium with perfect market models is an "accident," in that it requires special combinations of parameters of the system that are "negligible" in the whole parameter space, in a suitable sense. This situation may be summarized by the statement that "existence of rational expectations equilibrium is generic."

The proof of generic existence that I shall present demonstrates in addition a remarkable information efficiency property of equilibrium. Generically, in the model considered here, a rational expectations equilibrium reveals to all traders the information possessed by all of the traders taken together. ${ }^{3}$ Seen in a broader context, this property of equilibrium might cast doubt on the incentives for a trader to obtain information about the environment prior to entering the market, provided he could count on other traders obtaining the same information, which would then be revealed by market prices. But if each trader reasoned in this way, then no trader would obtain prior information, and so there would be no prior information for market prices to reveal! However, to examine this question more carefully one needs a model that reflects the dynamics of market adjustment and price formation. ${ }^{4}$

Another approach would be to explore the case in which traders have models of market price determination that are imperfect or imprecise, but that are consistent with observations of the market during the process of model revision or "learning." This approach will be followed in a subsequent paper. ${ }^{5}$

The concept of rational expectations equilibrium has also received considerable attention in the macroeconomic literature (see Shiller [15] for a review). However, no attempt will be made here to relate the present paper to that literature.

The proof of the main result of the present paper, on the generic existence and informational efficiency of rational expectations equilibrium, is based on an auxiliary proposition that has some independent interest. Roughly speaking, this auxiliary proposition concerns the comparison of ordinary exchange equilibria under uncertainty in which traders have (subjective) probability beliefs about the

\footnotetext{
${ }^{2}$ Nonexistence of rational expectations equilibrium may be caused by a discontinuity in the market demand functions that can arise when traders use market prices to infer something about other traders' information. This discontinuity was pointed out in Radner [13], where conditions for the Pareto optimality of rational expectations equilibrium were discussed. Specific examples of nonexistence of equilibrium have been given in Green [4] and Kreps [11]; a further discussion of existence can be found in Jordan $[7,8]$.

${ }^{3}$ Conditions under which equilibrium prices reveal traders' initial information have been explored in Green [3], Grossman [5], Grossman and Stiglitz [6], and Kihlstrom and Mirman [10].

${ }^{4}$ See Beja [1] for a step in this direction.

${ }^{5}$ See Radner [14].
} 
payoff-relevant state of the environment. The proposition gives conditions under which, generically, two exchange economies that differ only in the traders' probability distributions will have different equilibrium prices. Since the argument leading to the main result (Section 3 ) is fairly long, I shall give a heuristic sketch in this introductory section.

\section{Pure Exchange under Uncertainty}

Consider first a pure exchange economy. The decision problem for trader $i$ is to choose a vector of assets, which will be called his portfolio. The eventual utility to $i$ of his portfolio is uncertain at the time he purchases it. This is expressed by saying that his utility depends on his portfolio and on the environment (which is exogenous). Each trader has a subjective probability distribution on the set of alternative environments, and we suppose that his criterion for choosing among alternative portfolios is expected utility. Given his initial endowment of assets, and given a vector of asset prices, he will demand a portfolio that maximizes his expected utility subject to the budget constraint that the cost of his portfolio not exceed the value of his initial endowment. For simplicity, I shall suppose that only nonnegative portfolios are allowed (no short sales). His excess demand is the vector of differences between the assets he demands and his initial endowments. Suppose that his utility function is sufficiently regular so that, for any vector of prices, his excess demand is unique. Let $p$ denote the vector of assets prices, let $\pi$ denote the array of subjective probability distributions of the environment (one for each trader), and let $Z(p, \pi)$ denote the corresponding total excess demand, i.e. the (vector) sum of the individual traders' excess demands. Given a probability array $\pi$, an equilibrium is a price vector for which the total excess demand is zero, i.e. a solution $p$ of the equation system

$$
Z(p, \pi)=0 .
$$

With sufficient regularity conditions on the traders' utility functions, at least one equilibrium will exist for every probability array.

The auxiliary proposition deals with the question, under what conditions must two different probability arrays lead to different corresponding equilibria? Call a pair $\left(\pi_{1}, \pi_{2}\right)$ of probability arrays confounding if there is a solution $\left(p_{1}, p_{2}\right)$ of the equation system

$$
\begin{aligned}
& Z\left(p_{1}, \pi_{1}\right)=0, \\
& Z\left(p_{2}, \pi_{2}\right)=0, \\
& p_{1}=p_{2} .
\end{aligned}
$$

In other words, for a confounding pair of probability arrays, there exists a single price vector that is an equilibrium for each of them. On the other hand, if a pair of probability arrays is not confounding, then any corresponding pair of equilibrium price vectors will be distinct. 
It is easy to give examples of "textbook" utility functions for which confounding pairs of probability arrays exist (see Section 2). However, since the equation system (1.2) has more equations than unknowns, it would seem plausible that, if the equations were in "general position" then no solution would exist. To make this idea precise, assume that the set $E$ of alternative states of the environment is finite; then a probability distribution on $E$ can be represented as a point in a vector space of dimension $\# E-1$, where $\# E$ denotes the number of states in $E$ (recall that the probabilities must sum to unity). A probability array can be represented by a point in a space of dimension $I(\# E-1)$, where $I$ is the number of traders, and the set $\Pi_{0}$ of pairs of probability arrays lies in a space of dimension $2 I(\# E-1)$. The equation system (1.2) is thus parameterized by points in $\Pi_{0}$.

Since asset prices are relative, it will be understood that they are to be normalized, say by taking the sum to be unity. Hence, if there are $n$ assets, there are $(n-1)$ independent prices, so $(1.2)$ has $2(n-1)$ unknowns (for every parameter point in $\left.\Pi_{0}\right)$. On the other hand, there are nominally $3 n$ equations in (1.2). However, if the utility functions are such that every budget is exhausted, then the value of excess demand will always be zero, even out of equilibrium (Walras's law). Furthermore, as noted above, there are only $(n-1)$ independent prices, so the condition $p_{1}=p_{2}$ represents only $(n-1)$ independent equations. Hence there are at most $3(n-1)$ independent equations in (1.2). This is still larger than the number of unknowns, however, so that one would not typically expect (1.2) to have a solution.

Call a subset of $\Pi_{0}$ negligible if its closure has Lebesgue measure zero. The auxilliary proposition gives conditions under which the set of confounding pairs of probability arrays is negligible. In terms of the equation system (1.2), this conclusion can be interpreted as follows. Let $C_{0}$ be the set of confounding pairs in $\Pi_{0}$, and let $\bar{C}_{0}$ be the closure of $C_{0}$ in $\Pi_{0}$. If a parameter point is in $C_{0}$ (i.e. confounding), then every neighborhood of it has a parameter point for which (1.2) has no solution. In other words, for a parameter point in $C_{0}$ there exist arbitrarily small perturbations of the system (1.2), i.e. arbitrarily small perturbations of the parameter point, for which the equations have no solution. On the other hand, if a parameter point is not in $\bar{C}_{0}$ (and is therefore not confounding), then there is some neighborhood of it such that for all points in the neighborhood the system (1.2) has no solution. (In addition, there may be points of $\bar{C}_{0}$ that are not confounding, i.e. not in $C_{0}$.)

In another terminology, if a property holds except on a negligible set, one says that it holds generically. In this terminology, the conclusion of the auxillary proposition is that, generically, different probability arrays give rise to different equilibrium prices.

\section{Full Communication Equilibria and Revealing Prices}

At the next stage in the analysis, I introduce the concepts of full communication equilibrium and revealing prices. Consider a pure exchange situation similar to the one just described, except that before the market activity takes place some exogenous information about the environment is made available to all the traders. 
To express the idea that this information may be incomplete or noisy, let $s$ denote the information signal, and suppose that every trader has a subjective joint probability distribution of the signal $s$ and the environment $e$. (Strictly speaking, since the exogenous information signal $s$ is also part of the traders' environment, one should now call $e$ the payoff-relevant part of the environment (see J. Marschak [12]), but for simplicity I shall continue to call $e$ the environment.) Given the information signal $s$, each trader's preferences among portfolios will be determined by his conditional expected utility, using his conditional probability distribution of $e$ given $s$. Denote trader $i$ 's conditional distribution of $e$ given $s$ by (the vector) $\pi_{s i}$, and for each $s$ denote the array $\left(\pi_{s i}\right)$ of distributions, one for each trader, by $\pi_{s}$. Corresponding to each signal $s$, an equilibrium price vector, $p_{s}$, is a solution of the equation system

$$
Z\left(p_{s}, \pi_{s}\right)=0 .
$$

A full communication equilibrium (FCE) is mapping, $\hat{\phi}$, from information signals to price vectors such that, for each signal $s, \hat{\phi}(s)$ is an equilibrium for $s$, i.e. a solution $p_{s}$ of (1.3). (The reason for the terminology "full communication" will become apparent later.) I shall say that a FCE is revealing if it is one-to-one, i.e. it maps different signals into distinct price vectors. (Recall that all price vectors are normalized.) Thus if market prices are determined by a revealing FCE, then one can infer the underlying signal from observing the market prices.

Assume that the set $S$ of alternative information signals is finite, and let $\pi$ denote the (finite) array of probability arrays $\pi_{s}$. The set $\Pi$ of arrays $\pi$ has dimension $(\# S) I(\# E-1)$. Also, if $S$ is finite, the function $\hat{\phi}$ is actually a finite-dimensional vector, with (\#S) $n$ coordinates (where $n$ is the number of assets); because of the normalization of prices, $\hat{\phi}$ in fact lies in a set of dimension $(\# S)(n-1)$. Thus a point $\pi$ in $\Pi$ is a vector of parameters for the system of equations

$$
Z\left[\hat{\phi}(s), \pi_{s}\right]=0, \quad \text { for every } s \text { in } S ;
$$

this is a system of finitely many equations in finitely many unknowns.

Observe that, for any parameter point $\pi$, every FCE is revealing if and only if, for every pair $\left(s, s^{\prime}\right)$ of distinct signals, the pair $\left(\pi_{s}, \pi_{s^{\prime}}\right)$ is not confounding. It is easy to check that, if a set $C_{0}\left(s, s^{\prime}\right)$ of pairs $\left(\pi_{s}, \pi_{s^{\prime}}\right)$ is negligible in the corresponding space of dimension $2 I(\# E-1)$, then the set $C\left(s, s^{\prime}\right)$ of points $\pi$ in $\Pi$ for which $\left(\pi_{s}, \pi_{s^{\prime}}\right)$ is in $C_{0}\left(s, s^{\prime}\right)$ is also negligible. Let $C$ be the set of points $\pi$ in $\Pi$ such that, for some distinct $s$ and $s^{\prime}$, the pair $\left(\pi_{s}, \pi_{s^{\prime}}\right)$ is confounding. Since the set $S$ is finite, and the union of finitely many negligible sets is negligible, it follows that, if for every distinct $s$ and $s^{\prime}$ the set of confounding pairs is negligible, then the set $C$ is also negligible. Hence the conclusion of the first auxillary proposition (above) implies that, generically, in $\Pi$, a full communication equilibrium is revealing.

\section{Differential Information and Rational Expections Equilibrium}

At the final stage in the analysis, I consider the situation in which different traders come to the market with possibly different exogenous information signals. 
Let $s_{i}$ denote the exogenous information signal available to trader $i$, and let $s=\left(s_{1}, \ldots, s_{I}\right)$ denote the total exogenous information available in the market. Thus each trader may have only a part of the total information available. Each trader $i$ has a subjective joint probability distribution of $s$ and $e$.

As a preliminary thought experiment, imagine that, given his information signal $s_{i}$, each trader chose among portfolios according to his conditional expected utility, using his conditional probability distribution of $e$ given $s_{i}$. This would generate an excess demand function for each trader $i$, given $s_{i}$, and thus would generate a total excess demand function for all traders, given $s$. An "exogenous information equilibrium" price vector, given $s$, would be one for which this total excess demand would be zero. For each $s$ let $\phi(s)$ be a corresponding exogenous equilibrium price vector, and suppose that every trader behaves in such a way that $\phi(s)$ is in fact the market price if $s$ is realized and each trader $i$ observes $s_{i}$.

Now imagine that, after a number of independent realizations of this situation, a particular trader, say number 1, becomes "sophisticated" and realizes that there is a regular relationship between the total information signal and the market price; this relationship is, of course, described by the mapping $\phi$. Trader 1 would then be able to infer something about the total information signals from his observations of the market price, $\phi(s)$ (unless, of course, the market price were the same for all $s)$. This would change his excess demand function, since the market price would not only enter his budget constraint, but would also provide a supplementary signal-in addition to his exogenous signal $s_{i} \multimap$ on which to condition his expected utility. But if his excess demand were not an insignificant part of the total, this "sophisticated" behavior would change the total excess demand function, too, which would change the relation $\phi$ between total exogenous information and market price! In fact, if all traders became sophisticated in this manner, then the original exogenous equilibrium price vectors $\phi(s)$ would typically no longer clear the market given the total exogenous information signal $s$. What would be required would be a "forecast function" $\phi$ that would be self-fulfilling.

These preliminary considerations motivate the following formal definitions. A forecast function $\phi$ is a mapping that associates with each total exogenous information signal $s$ a price vector $\phi(s)$. Given a forecast function $\phi$, suppose that each trader $i$ chooses among portfolios according to his conditional expected utility given the (augmented) information $\left[s_{i}, \phi(s)\right]$.

This behavior will generate, for each total signal and each trader an excess demand; call the resulting total excess demand the sophisticated excess demand. It should be emphasized that this sophisticated excess demand depends on the forecast function $\phi$ and on the particular total signal $s$; denote it by $\zeta(s, \phi)$. A rational expectations equilibrium (REE) is a forecast function such that the corresponding sophisticated excess demand is zero for every total information signal, i.e. a function $\phi$ such that

$$
\zeta(s, \phi)=0, \text { for all } s \text { in } S .
$$

Note that sophisticated demand behavior requires the knowledge of the entire function $\phi$ to determine the demand corresponding to any single signal $s$, and that 
the equation system (1.5) is a system of simultaneous equations in the values of the function $\phi$.

We can now see the connection between full communication and rational expectations equilibria. Consider a FCE $\hat{\phi}$ based on the total information signals. In our present terminology, the FCE $\hat{\phi}$ is a particular forecast function. Recall that $\hat{\phi}$ is revealing if it is one-to-one. Suppose that the FCE were revealing; then for every trader and every total information signal $s$, the conditional probability distribution of $e$ given $\hat{\phi}(s)$ would be the same as the conditional probability distribution of $e$ given $s$. Hence the sophisticated demand of trader $i$ given $s_{i}$ and $\hat{\phi}(s)$ would equal his ordinary demand given $s$. By the equilibrium property of $\hat{\phi}$, the ordinary total excess demand, given that every trader knows $s$ and that the price vector is $\hat{\phi}(s)$, is zero. It follows that the FCE $\hat{\phi}$ is a forecast function that satisfies (1.5). Thus we see that a revealing full communication equilibrium is $a$ rational expectations equilibrium.

One can now state the main result about REE's as a corollary of the preceding observation and the auxillary proposition: Under the assumptions of the auxillary proposition, generically, there exists a rational expectations equilibrium that is revealing.

In Section 2, I provide a simple example that illustrates the concepts and the main results. Section 3 contains a systematic presentation of all of the results and their proofs. In the course of proving the main results, it is necessary to prove that the excess demand function is generically differentiable in prices and parameters at equilibrium (Lemma 1); the method used may be applicable to other models. The assumptions are discussed in some detail in Section 4.

\section{AN EXAMPLE}

An example in which the equilibrium can be explicitly calculated illustrates the problems that will be considered in subsequent sections. ${ }^{6}$

Suppose that there are only two assets being traded. Trader $i$ 's initial endowment will be denoted by $w_{i}=\left(t_{i}, v_{i}\right)$ and his final portfolio by $x_{i}=\left(y_{i}, z_{i}\right)$. The (normalized) price vector is $p=(q, 1-q)$. Suppose further that trader $i$ 's utility function is of the "Cobb-Douglas" form:

$$
u_{i}\left(x_{i}, \alpha_{i}\right) \equiv \alpha_{i} \log y_{i}+\left(1-\alpha_{i}\right) \log z_{i}, \quad 0<\alpha_{i}<1
$$

First consider the case of equilibrium with certainty. It is easily verified that, if trader $i$ chooses $x_{i}$ to maximize the utility (2.1) subject to

$$
\begin{aligned}
& y_{i} \geqslant 0, \quad z_{i} \geqslant 0, \\
& q y_{i}+(1-q) z_{i} \leqslant q t_{i}+(1-q) \dot{v}_{i},
\end{aligned}
$$

\footnotetext{
${ }^{6}$ Strictly speaking, this example is not a special case of the model of Section 3 . However, the simplicity of the formulas for equilibrium prices makes it useful for exposition.
} 
then his demand, as a function of the price vector, is

$$
\begin{aligned}
& y_{i}=\frac{\alpha_{i} W_{i}}{q}, \quad z_{i}=\frac{\left(1-\alpha_{i}\right) W_{i}}{1-q}, \\
& W_{i} \equiv q t_{i}+(1-q) v_{i} .
\end{aligned}
$$

For an equilibrium, excess demand equals zero:

$$
\sum_{i}\left(y_{i}-t_{i}\right)=\sum_{i}\left(z_{i}-v_{i}\right)=0 .
$$

The solution, for $q$, of (2.3) and (2.4) is

$$
q=\frac{\sum_{i} \alpha_{i} v_{i}}{\sum_{i}\left[\alpha_{i} v_{i}+\left(1-\alpha_{i}\right) t_{i}\right]}
$$

Now suppose that there are two alternative states of the environment, with respective probabilities $\psi^{\prime}$ and $\psi^{\prime \prime} ; \psi^{\prime}+\psi^{\prime \prime}=1$. The parameter of trader $i$ 's utility function may depend on the state of the environment; let $\alpha_{i}^{\prime}$ and $\alpha_{i}^{\prime \prime}$ denote the two respective values of $\alpha_{i}$. If trader $i$ does not know the state of the environment when he makes his purchase decision, then he will maximize his expected utility

$$
u_{i}\left(x_{i}, \bar{\alpha}_{i}\right)=\bar{\alpha}_{i} \log y_{i}+\left(1-\bar{\alpha}_{i}\right) \log z_{i},
$$

where

$$
\bar{\alpha}_{i} \equiv \psi^{\prime} \alpha_{i}^{\prime}+\psi^{\prime \prime} \alpha_{i}^{\prime \prime} .
$$

I now consider three types of equilibrium. First, if every trader knows the state of the environment when he makes his purchase decision, then in each state there will be an equilibrium. Denote the respective equilibrium prices by $\hat{q}^{\prime}$ and $\hat{q}^{\prime \prime}$; these are obtained from (2.5) by letting $\alpha_{i}$ equal $\alpha_{i}^{\prime}$ and $\alpha_{i}^{\prime \prime}$, respectively. Call these the full communication equilibrium prices.

Second, let $(J, K)$ be a partition of the traders into two sets, such that at the time of purchase, traders in $J$ (the "informed" traders) know the state of the environment, and traders in $K$ (the "uninformed" traders) do not. The resulting equilibrium prices in the two states will be

$$
\tilde{q}^{\prime}=\frac{\sum_{i \in J} \alpha_{i}^{\prime} v_{i}+\sum_{i \in K} \bar{\alpha}_{i} v_{i}}{\sum_{i \in J}\left[\alpha_{i}^{\prime} v_{i}+\left(1-\alpha_{i}^{\prime}\right) t_{i}\right]+\sum_{i \in K}\left[\bar{\alpha}_{i} v_{i}+\left(1-\bar{\alpha}_{i}\right) t_{i}\right]},
$$

$$
\tilde{q}^{\prime \prime}=\frac{\sum_{i \in J} \alpha_{i}^{\prime \prime} v_{i}+\sum_{i \in K} \bar{\alpha}_{i} v_{i}}{\sum_{i \in J}\left[\alpha_{i}^{\prime \prime} v_{i}+\left(1-\alpha_{i}^{\prime \prime}\right) t_{i}\right]+\sum_{i \in K}\left[\bar{\alpha}_{i} v_{i}+\left(1-\bar{\alpha}_{i}\right) t_{i}\right]} .
$$


Call these the unsophisticated equilibrium prices. Note that this includes the full communication equilibrium as a special case, when $K$ is empty.

To introduce the third equilibrium concept, imagine that in the process of approaching the second equilibrium by means of a tâtonnement process, the uninformed traders realize that the equilibrium price should reflect the information that the informed traders possess. The possibility of inferring something about the informed traders' information from the market price rests, of course, on knowing how the market price would differ in the two states of the environment, but this relationship is endogenous to the market system. Thus, define the third equilibrium as a pair of prices, say $\left(q^{\prime}, q^{\prime \prime}\right)$, such that if every trader expects $q^{\prime}$ to be the market price in state 1 and $q^{\prime \prime}$ to be the price in state 2 , and if each trader maximizes his conditional expected utility, conditioned on both his own initial information and the market price, then excess demand will be zero in each state. Call this a rational expectations equilibrium.

In this example there are two possibilities for a rational expectations equilibrium $\left(q^{\prime}, q^{\prime \prime}\right)$ : either (i) $q^{\prime}$ and $q^{\prime \prime}$ are unequal, or (ii) they are equal. In the first case initially uninformed traders (those in $K$ ) can infer the state of the environment from the market price, so that, conditional on the market price, all traders become informed. In this case, I shall say that the equilibrium prices are revealing. Therefore in case (i)

$$
q^{\prime}=\hat{q}^{\prime}, \quad q^{\prime \prime}=\hat{q}^{\prime \prime}, \quad \hat{q}^{\prime} \neq \hat{q}^{\prime \prime} ;
$$

the equilibrium prices must be the same as those in the first, full communication equilibrium.

In the second case, initially uninformed traders cannot infer the state of the environment from the market price, so that, conditional on the market price, each trader's information is the same as in the unsophisticated equilibrium, (2.8). Thus case (ii) is characterized by

$$
q^{\prime}=\tilde{q}^{\prime}, \quad q^{\prime \prime}=\tilde{q}^{\prime \prime}, \quad \tilde{q}^{\prime}=\tilde{q}^{\prime \prime} .
$$

Which of these two cases is possible (if any) depends on the parameters of the model, $\left(\alpha_{i}^{\prime}\right),\left(\alpha_{i}^{\prime \prime}\right), \psi^{\prime}$, and $(J, K)$, which in turn determine $\hat{q}^{\prime}, \hat{q}^{\prime \prime}, \tilde{q}^{\prime}$, and $\tilde{q}^{\prime \prime}$. The possible rational expectations equilibria are summarized in the two-way table (Table I). The four cells of the table are labelled with Roman numerals. A rational

TABLE I

\begin{tabular}{|c|c|c|}
\hline & $\tilde{q}^{\prime} \neq \tilde{q}^{\prime \prime}$ & $\tilde{q}^{\prime}=\tilde{q}^{\prime \prime}$ \\
\hline$\hat{q}^{\prime} \neq \hat{q}^{\prime \prime}$ & $\begin{array}{c}\left(\hat{q}^{\prime}, \hat{q}^{\prime \prime}\right) \\
(\mathrm{I})\end{array}$ & $\begin{array}{c}\left(\hat{q}^{\prime}, \hat{q}^{\prime \prime}\right) \\
\text { and } \\
\left(\tilde{q}^{\prime}, \tilde{q}^{\prime \prime}\right) \\
(\mathrm{II})\end{array}$ \\
\hline$\hat{q}^{\prime}=\hat{q}^{\prime \prime}$ & $\begin{array}{l}\text { none } \\
\text { (III) }\end{array}$ & $\begin{array}{c}\left(\tilde{q}^{\prime}, \tilde{q}^{\prime \prime}\right) \\
(\mathrm{IV})\end{array}$ \\
\hline
\end{tabular}

RATIONAL EXPECTATIONS EQUILIBRIA 
expectations equilibrium exists in all cases except III, and is unique in I and IV.

If we fix the partition $(J, K)$ of agents into initially informed and uninformed traders, then there are $(2 I+1)$ remaining (real-valued) parameters, all of which are constrained to be between 0 and 1 . Cases II and III each correspond to a single equation and a single inequality, and case IV corresponds to two equations. For example, case III corresponds to the equality

$$
\frac{\sum_{i} \alpha_{i}^{\prime} v_{i}}{\sum_{i}\left[\alpha_{i}^{\prime} v_{i}+\left(1-\alpha_{i}^{\prime}\right) t_{i}\right]}=\frac{\sum_{i} \alpha_{i}^{\prime \prime} v_{i}}{\sum_{i}\left[\alpha_{i}^{\prime \prime} \cdot v_{i}+\left(1-\alpha_{i}^{\prime \prime}\right) t_{i}\right]}
$$

(cf. (2.5)), together with the inequality

$$
\tilde{q}^{\prime} \neq \tilde{q}^{\prime \prime},
$$

which I do not write out in full (cf. (2.8)). Equation (2.11) determines, in general, a manifold of dimension $2 I$ in the parameter space, and inequality (2.12) excludes from that manifold a submanifold of dimension $(2 I-1)$. Thus case III corresponds to a subset of the parameter space that essentially has dimension $2 I$, one less than the dimension of the entire parameter space. Continuing in this way, we may describe the four regions in the parameter space, corresponding to the four cells in Table I, as in Table II.

\begin{tabular}{|c|c|c|c|}
\hline Case & Conditions & Dimension & $\begin{array}{c}\text { Rational Expectations } \\
\text { Equilibria }\end{array}$ \\
\hline I & $\begin{array}{l}\hat{q}^{\prime} \neq \hat{q}^{\prime \prime} \\
\tilde{q}^{\prime} \neq \tilde{q}^{\prime \prime}\end{array}$ & $2 I+1$ & 1 \\
\hline II & $\begin{array}{l}\hat{q}^{\prime} \neq \hat{q}^{\prime \prime} \\
\tilde{q}^{\prime}=\tilde{q}^{\prime \prime}\end{array}$ & $2 I$ & 2 \\
\hline III & $\begin{array}{l}\hat{q}^{\prime}=\hat{q}^{\prime \prime} \\
\tilde{q}^{\prime} \neq \tilde{q}^{\prime \prime}\end{array}$ & $2 I$ & 0 \\
\hline IV & $\begin{array}{l}\hat{q}^{\prime}=\hat{q}^{\prime \prime} \\
\tilde{q}^{\prime}=\tilde{q}^{\prime \prime}\end{array}$ & $2 I-1$ & 1 \\
\hline
\end{tabular}

TABLE II

Thus, except for a closed set of Lebesque measure zero in the parameter space, a rational expectations equilibrium (REE) exists, is unique, and is identical in outcomes with the full communication equilibria. The sets for which no REE exists (III) and two REE exist (IV) are each of dimension one less than that of the parameter space, and the set for which the unique equilibrium does not reveal the state of the environment to the initially uninformed traders is "least likely" of all, having dimension two less than that of the parameter space.

In a more general setting, each trader would have some initial information about the environment, and the market price would depend at most on the initial information available jointly to all the traders. Corresponding to the above full 
communication equilibria would be equilibria in which all traders were provided in common all the information that was jointly available to them initially; call these also full communication equilibria. The analysis of this example suggests the conjecture $^{7}$ that, under much more general conditions, a REE will have the same outcomes as the full communication equilibria, except for a "small" set in the space of parameters of the market system. This conjecture is proved in Section 3, in the context of a more general model, under the condition that the set of alternative states of initial information is finite.

\section{RATIONAL EXPECTATIONS AND FULL COMMUNICATION EQUILIBRIA}

In this section I show that, in the context of a particular model of asset trading, the existence of a rational expectations equilibrium that is also a revealing full communication equilibrium is generic. In other words, except for a negligible set of points in the parameter space of the economy, rational expectations equilibria exist that reveal to all traders all the available information.

Consider a pure exchange economy with $I$ traders. Trader $i$ chooses $\left(c_{i}, z_{i}\right)$, where $c_{i}=i$ 's expenditure on current consumption, and $z_{i}=i$ 's portfolio, with $K$ different assets. Thus $c_{i}$ is a nonnegative real number, and $z_{i}$ is a nonnegative $K$-dimensional vector. The value of one unit of asset $k$ next period will be $v^{k}$; however, at the time of the current market traders are uncertain about the vector $v=\left(v^{k}\right)$ of future asset values. The vector $v$ can take on one of finitely many values $v_{e}, e$ in $E$; call $e$ the payoff-relevant environment. If trader $i$ chooses $(c, z)$, and environment $e$ obtains, then the future value of his portfolio will be the inner product ${ }^{8} v_{e}^{\prime} z$; assume that the utility of this outcome to him is $U_{0 i}(c)+U_{i}\left(v_{e}^{\prime} z\right)$. We may interpret $U_{i}(y)$ as the indirect utility of starting next period with wealth $y$.

Before making any trade, each trader $i$ has available exogenously some information, $s_{i}$, a point in a finite set $S_{i}$. I shall call $s_{i}$ the signal received by trader $i$, and the $I$-tuple $s=\left(s_{1}, \ldots, s_{I}\right)$ the joint signal. Let $S$ denote the (finite) set of all joint signals. Each trader $i$ has a probability distribution on $E \times S$. Different traders may have different beliefs about the joint distribution $Q_{i}$ of $e$ and $s$.

I shall later postulate conditions that will guarantee that at equilibrium all prices are positive. Therefore, it is legitimate to normalize prices so that the price of current consumption is 1 ; let $q$ denote the vector of asset prices.

Finally, let $\omega_{i}$ denote trader $i$ 's vector of initial endowments. We may interpret the first coordinate of $\omega_{i}$ as $i$ 's endowment of "cash." If the coordinates of $\omega_{i}$ are numbered $0, \ldots, K$, and $w_{i} \equiv\left(\omega_{i}^{1}, \ldots, \omega_{i}^{K}\right)$ (i's asset endowment), then trader $i$ 's budget constraint is

$$
c_{i}+q^{\prime} z_{i} \leqslant \omega_{i}^{0}+q^{\prime} w_{i} \equiv W_{i}
$$

\footnotetext{
${ }^{7}$ As far as I know, this idea is essentially due to Michael Rothschild.

${ }^{8}$ Vectors will ordinarily be understood to be columns, and their transpose will be denoted by a prime. $N$-dimensional Euclidean space will be denoted by $R^{N}$, its nonnegative orthant by $R_{+}^{N}$, and its strictly positive orthant by $R_{++}^{N}$.
} 
Before formally specifying each trader's behavior, let $\xi_{i s_{i}}(q)$ provisionally denote trader $i$ 's demand, given the asset price vector $q$, and his signal $s_{i}$, and let $Z_{s}(q)$ provisionally denote the total market excess demand for assets. Suppose that every trader's demand satisfies his budget constraint (3.1) with equality; then, by Walras's Law, zero excess demand for assets implies zero excess demand for current consumption. Therefore, $q$ is an equilibrium (asset) price vector, given $s$, if $Z_{s}(q)=0$.

Suppose that there were a rule for choosing a single equilibrium $q$ in the case of multiple equilibria; then for every $s$ there would be a well-defined equilibrium price vector, say $q_{s}=\phi(s)$. In this circumstance, any trader knowing the function $\phi$ would be able to infer that $s$ is in the inverse image, $\phi^{-1}(q)$, of $q$, and might be expected to evaluate his expected utility conditional both on $s_{i}$ and on $\sin \phi^{-1}(q)$.

This motivates the following definition of rational expectations equilibrium. Call a function from $S$ to $R_{+}^{K}$ a forecast function. Given a forecast function $\phi$, and given a price vector $q$ and a joint signal $s$, trader $i$ 's demand is a $(c, z)$ that maximizes his conditional expected utility

$$
\mathscr{E}_{Q_{i}}\left\{U_{i 0}(c)+U_{i}\left(v_{e}^{\prime} z\right) \mid s_{i}, s \text { in } \phi^{-1}(q)\right\},
$$

subject to the budget constraint (3.1). Let $\xi_{i s_{i}}(q, \phi)$ denote $i$ 's demand for assets; the total excess demand for assets is

$$
Z_{s}(q, \phi) \equiv \sum_{i}\left[\xi_{i s_{i}}(q, \phi)-w_{i}\right] .
$$

$A$ rational expectations equilibrium (REE) is a forecast function $\phi^{*}$ such that

$$
Z_{s}\left[\phi^{*}(s), \phi^{*}\right]=0 \text {, every } s \text { in } S .
$$

Note that (3.4) is a set of simultaneous equations in all of the values of the forecast function $\phi^{*}$.

\section{Full Communication Equilibria}

I shall now propose a candidate for a rational expectations equilibrium. Imagine that before entering the market the traders exchange all their signal information, so that every trader knows $s$. Let $\hat{\phi}(s)$ denote an ordinary equilibrium price vector given $s$, i.e. a price vector for which excess demand would be zero if each trader maximized conditional expected utility given $s$. Call $\hat{\phi}$ a full communication equilibrium $(F C E)$. (Actually, $\hat{\phi}$ is a family of equilibrium price vectors, one for each joint signal $s$.) Call $\hat{\phi}$ revealing if different joint signals $s$ result in different equilibrium price vectors $\hat{\phi}(s)$, i.e.

$$
s \neq s^{\prime} \text { implies } \hat{\phi}(s) \neq \hat{\phi}\left(s^{\prime}\right) .
$$

It is immediate that if $\hat{\phi}$ is a FCE and satisfies (3.5), then it satisfies (3.4). Thus, $a$ full communication equilibrium that is revealing is a rational expectations equilibrium. 
With this observation in mind, let us turn to the examination of conditions under which a FCE is revealing. For any trader $i$, any joint signal $s$, and environment $e$, let $\pi_{\text {ise }}$ denote $i$ 's conditional probability of $e$, given $s$; let $\pi_{i s}$ denote the probability vector with coordinates $\left(\pi_{i s e}\right)$; let $\pi_{s}$ denote the array $\left(\pi_{i s}\right)$, $i=1, \ldots, I$; and let $\pi$ denote $^{9}$ the array $\left(\pi_{s}\right)$. Given $s$, trader $i$ 's conditional expected utility of the pair $(c, z)$ is

$$
U_{0 i}(c)+\sum_{e} \pi_{i s e} U_{i}\left(v_{e}^{\prime} z\right)
$$

Therefore, in a FCE, trader $i$ 's conditional expected utility of $(c, z)$ given $s$ depends on $s$ only through the probability vector $\pi_{i s}$, and total excess demand in the market depends on $s$ only through the array $\pi_{s}$. To emphasize this, I shall denote this excess demand by $Z\left(q, \pi_{s}\right)$.

With this notation, a FCE is a solution, $\left(q_{s}\right)$, of the following system of equations:

$$
Z\left(q_{s}, \pi_{s}\right)=0, \text { for all } s \text { in } S .
$$

(Note that, for each $s$, the equation for $q_{s}$ can be solved by itself.) The FCE is revealing if all of the price vectors $q_{s}$ are distinct.

Let $\Pi$ denote the set of all probability arrays $\pi=\left(\pi_{s}\right)$. Call a subset $C$ of $\Pi$ negligible if the closure of $C$ has Legesgue measure zero in $\Pi$. I wish to show that the set of points $\pi$ in $\Pi$ for which there is no corresponding revealing FCE is negligible. It will then follow that, except for a negligible set of points in $\Pi, a$ revealing rational expectations equilibrium exists. Actually I shall prove a somewhat stronger statement about FCE's, namely, that the set of points in $\Pi$ for which there exists some nonrevealing FCE is negligible.

The proof rests on three assumptions, which I now introduce and motivate.

The first two assumptions guarantee that full communication equilibria exist, that demands are single-valued (as opposed to set-valued), and that in equilibrium every trader's current consumption is strictly positive. ${ }^{10}$

(A1) For every trader $i, U_{0 i}$ and $U_{i}$ are twice continuously differentiable, strictly increasing, and strictly concave; furthermore, $U_{0 i}^{\prime}(c)$ tends to $+\infty$ as $c$ tends to zero.

(A2) For every $i, \omega_{i}>0$; further, $\Sigma_{i} \omega_{i} \gg 0$.

The third assumption concerns the (future) values of the alternative assets in the several states of the environment. Part (a) requires that there be more states of the environment than there are assets, that the set of future-value vectors $v_{e}$ be sufficiently varied, and that every asset have a strictly positive future value in every state. Part (b) rules out the situation in which the marginal utility of a small change in a portfolio would be the same in all states (at equilibrium).

\footnotetext{
${ }^{9}$ Warning: the symbol $\pi$ will always be used to denote an array of probability vectors, but at different points in the paper the arrays may be of different dimension. The proper dimension of $\pi$ will, of course, be indicated in each context.

${ }^{10}$ For a vector or matrix $x, x \geqslant 0$ means that every element of $x$ is nonnegative; $x>0$ means that $x \geqslant 0$ and $x \neq 0 ; x \gg 0$ means that every element of $x$ is strictly positive.
} 
(A3) (a) $E$ has more than $K$ elements $\left(\# E>K\right.$ ), every set of $K$ vectors $v_{e}$ spans $R^{K}$, and every $v_{e} \gg 0$; (b) at equilibrium, for every $i$, there exists no $x$ in $R^{K}$ such that for every $e\left(v_{e}^{\prime} x\right) U_{i}^{\prime}\left(v_{e}^{\prime} z_{i}\right)=1$.

In particular part (b) of (A3) rules out the logarithmic utility function $U_{i}(y)=$ $\log y$. It will be shown to follow from part (a) that the expected utility function is strictly concave in the portfolio vector $z$.

\section{Equilibrium under Uncertainty is Sensitive to the Probabilities}

The proof that, generically, a FCE is revealing can be based on an auxillary proposition about ordinary equilibrium under uncertainty, which has some independent interest. Let $\pi_{1}$ and $\pi_{2}$ be two probability arrays in $P^{I}$ where $P$ denotes the set of all probability vectors $\left(p_{e}\right)$, i.e. the set of all probability distributions on $E$. Looking at the system of equations (3.7), one sees that one wants conditions under which the following system has no solution:

$$
\begin{aligned}
& Z\left(q_{1}, \pi_{1}\right)=0, \\
& Z\left(q_{2}, \pi_{2}\right)=0, \\
& q_{1}=q_{2} .
\end{aligned}
$$

A pair $\left(\pi_{1}, \pi_{2}\right)$ for which (3.8) does have a solution will be called confounding. In other words, if two probability arrays are not confounding, then in any pair of corresponding equilibria the equilibrium price vectors are distinct.

PROPOSITION: If assumptions $(A 1)-(A 3)$ are satisfied, then the set of confounding pairs $\left(\pi_{1}, \pi_{2}\right)$ is negligible in $P^{2 I}$.

Proof: First note that the set $P_{+}$of strictly positive points in $P$ is open and has full measure in $P$. Hence it is sufficient to show that the set $C_{0}$ of confounding points in $P_{+}^{2 I}$ is negligible in the following sense: $C_{0}$ has Legesgue measure zero in $P_{+}^{2 I}$, and is relatively closed in $P_{+}^{2 I}$ (the intersection of $C_{0}$ with any closed subset of $P_{+}^{2 I}$ is closed).

I next investigate some properties of an individual trader's demand. For a trader with probability vector $\left(p_{e}\right)$, his expected utility is (I suppress the index $i$ ):

$$
U_{0}(c)+\sum_{e} p_{e} U\left(v_{e}^{\prime} z\right) .
$$

Here $U_{0}(c)$ is the utility from current consumption, and

$$
\bar{U}(z) \equiv \sum_{e} p_{e} U\left(v_{e}^{\prime} z\right)
$$

is the expected utility from the portfolio.

The vector of first derivatives and the matrix of second derivatives of $\bar{U}$ are, 
respectively,

$$
\begin{aligned}
& D \bar{U}(z)=\sum_{e} p_{e} U^{\prime}\left(v_{e}^{\prime} z\right) v_{e} \\
& D^{2} \bar{U}(z)=\sum_{e} p_{e} U^{\prime \prime}\left(v_{e}^{\prime} z\right) v_{e} v_{e}^{\prime} .
\end{aligned}
$$

First, since $U^{\prime}>0$ and, for every $e, p_{e}>0$ and $v_{e} \gg 0$,

$$
D \bar{U}(z) \gg 0 \text {. }
$$

Second, since $U^{\prime \prime}<0$ and the vectors $v_{e} \operatorname{span} R^{K}$,

$$
D^{2} \bar{U}(z) \ll 0 \text { and is negative definite. }
$$

Also, both $D \bar{U}$ and $D^{2} \bar{U}$ are continuous.

By (3.12) and (3.13), $\bar{U}$ is strictly increasing and strictly concave in $z$. Also, $U_{0}$ is strictly increasing and strictly concave in $c$. Hence, for any given positive prices, a trader's demand will be unique, and the budget constraint will be satisfied with equality.

I now turn to the study of the properties of total excess demand. I normalize the price of consumption to be unity. Since every individual trader's budget constraint is satisfied with equality, the value of total excess demand for assets and current consumption is zero, and an equilibrium for the probability array $\pi$ in $P^{I}$ is characterized by $Z(q, \pi)=0$. By standard methods one can show that equilibrium exists. In equilibrium, all asset prices will be strictly positive.

With the present model, an individual's demand function need not be everywhere differentiable in prices and probabilities. By Assumption (A2), at equilibrium the total demand for every asset is positive, but a particular trader's demand for a particular asset could be zero. In such a case, his demand for that asset would typically have a discontinuity in the derivative with respect to prices and/or probabilities at a point at which the demand for that asset just falls to zero. We shall see that the set of points in $P_{+}^{I}$ at which this can happen in equilibrium is negligible.

LEMMA 1: There is an open subset $\mathscr{D}$ of $P_{+}^{I}$ whose complement in $P_{+}^{I}$ has Legesgue measure zero, and such that, for every $\pi$ in $\mathscr{D}$ and every corresponding equilibrium price vector $q$, the excess demand function $Z$ is continuously differentiable in both arguments in a neighborhood of $(q, \pi)$.

(The proof of Lemma 1 will be deferred to the end of this section.)

To continue with the proof of the Proposition, define the mapping $F$ from $L \equiv R_{++}^{2 K} \times \mathscr{D}^{2}$ to $R^{3 K}$ by

$$
F\left(q_{1}, q_{2}, \pi_{1}, \pi_{2}\right) \equiv\left[\begin{array}{c}
Z\left(q_{1}, \pi_{1}\right) \\
Z\left(q_{2}, \pi_{2}\right) \\
q_{1}-q_{2}
\end{array}\right]
$$


Let $M$ be the set points $\left(q_{1}, q_{2}, \pi_{1}, \pi_{2}\right)$ in $L$ at which $F$ is zero. In terms of $M$, a pair $\left(\pi_{1}, \pi_{2}\right)$ in $\mathscr{D}^{2}$ is confounding if and only if there exists $\left(q_{1}, q_{2}\right)$ such that $\left(q_{1}, q_{2}, \pi_{1}, \pi_{2}\right)$ is in $M$. In other words, the set of confounding pairs $\left(\pi_{1}, \pi_{2}\right)$ in $\mathscr{D}^{2}$ is the coordinate projection of $M$ in $\mathscr{D}^{2}$.

By Lemma 1, $F$ is continuously differentiable in an open set, say $L^{0}$, containing $M$.

LemMa 2: At every point of $M$, the Jacobian $J$ of $F$ has rank $3 K$.

(The proof of Lemma 2 will be deferred to the end of this section.)

To continue with the proof of the Proposition, note that the dimension of $\mathscr{D}^{2}$ is $d \equiv 2 I(\# E-1)$, and so the dimension of $L^{0}$ is $2 K+d$. Hence, by Lemma $2, M$ is a differentiable manifold ${ }^{11}$ of dimension $(2 K+d-3 K)=d-K$. Let $T$ be the coordinate-projection mapping from $M$ into $\mathscr{D}^{2}$. Since $T$ is differentiable, and the dimension of $M$ is strictly less then the dimension of $\mathscr{D}^{2}$, the image $T(M)$ under $T$ has measure zero ${ }^{12}$ in $\mathscr{D}^{2}$. One can show that there is a compact subset, say $Q$, of $R_{++}^{21}$ such that $M$ is contained in $Q \times \mathscr{D}^{2}$. Since $Z$ is continuous on all of $L$, it follows easily that $T(M)$ is relatively closed in $\mathscr{D}^{2}$. Hence $T(M)$ is negligible in $\mathscr{D}^{2}$. But $T(M)$ is exactly the set of confounding pairs in $\mathscr{D}^{2}$. By Lemma $1, \mathscr{D}^{2}$ is open and of full measure in $P_{+}^{2 I}$. Hence the set of confounding pairs in $P^{2 I}$ is negligible in $P^{2 I}$. This completes the proof of the Proposition.

Recall that $\Pi$ is the set of all probability arrays $\left(\pi_{i s e}\right)$.

COROLLARY: Except for a negligible subset $C$ of $\Pi$, for every probability array in $\Pi$ every corresponding full communication equilibrium is revealing.

(I omit the proof of the Corollary; see the Introduction.)

The main theorem is now an immediate consequence of the Corollary and the observation that every revealing FCE is a REE.

THEOREM: Except for a negligible subset $C$ of $\Pi$, for every probability array in $\Pi$ there is a corresponding rational expectations equilibrium that is revealing.

Note that the Theorem does not exclude the possibility that there are points both in $C$ and outside it that have a nonrevealing REE.

Proof of Lemma 1: The technique is similar to that used to prove the Proposition. ${ }^{13}$ First we need a precise statement of conditions for equilibrium. For each trader let $\pi \equiv\left(\pi_{i e}\right)$ denote his vector of probabilities of payoff-relevant environments $e$. As before, let $q$ denote the vector of asset prices. Given $q \gg 0$,

\footnotetext{
${ }^{11}$ See Sternberg [16, Chapter II, p. 47, Exercise 3.5].

${ }^{12}$ See Sternberg [16, Chapter II, p. 47, Exercise 3.4].

${ }^{13}$ The idea of using this general method to prove the generic differentiability of the excess demand function was suggested to me by G. Chichilnisky. See also Chichilnisky and Kalman [2].
} 
each trader $i$ will use all of his budget, so his expected utility associated with a portfolio $z_{i}$ is

$$
V_{i}\left(z_{i}\right) \equiv U_{i 0}\left(\omega_{i}^{0}+q^{\prime} w_{i}-q^{\prime} z_{i}\right)+\sum_{e} \pi_{i e} U_{i}\left(v_{e}^{\prime} z_{i}\right),
$$

where $\omega_{i} \equiv\left(\omega_{i}^{0}, w_{i}\right)$ is his initial endowment. The constraints on his portfolio choice are:

$$
\begin{aligned}
& z_{i} \geqslant 0 . \\
& \omega_{i}^{0}+q^{\prime} w_{i}-q^{\prime} z_{i} \geqslant 0 .
\end{aligned}
$$

Since the marginal utility of period-zero consumption is infinite at zero consumption (A1), the second of the above constraints will not be binding. Hence, the first order conditions for a trader's demand are

$$
D V_{i}\left(z_{i}\right) \leqslant 0, \quad z_{i} \geqslant 0, \quad z_{i}^{\prime} D V_{i}\left(z_{i}\right)=0,
$$

where $D$ denotes the vector of first derivatives (these conditions are necessary and sufficient). Let $V_{i k}\left(z_{i}\right)$ denote the $k^{\prime}$ th coordinate of $D V_{i}\left(z_{i}\right)$; then an equivalent way of writing (3.18) is

$$
\begin{array}{lll}
z_{i} \geqslant 0, & & \\
z_{i}^{k}>0 & \text { implies } & V_{i k}\left(z_{i}\right)=0, \\
z_{i}^{k}=0 & \text { implies } & V_{i k}\left(z_{i}\right) \leqslant 0 .
\end{array}
$$

The demand $z_{i}^{k}$ can fail to be differentiable in $q$ and/or $\pi_{i}$ only in the case in which both $z_{i}^{k}=0$ and $V_{i k}\left(z_{i}\right)=0$.

For equilibrium, we add the condition that total excess demand for assets be zero:

$$
\sum_{i}\left(z_{i}-w_{i}\right)=0 .
$$

For any array $\left(\pi_{i}\right)$ in $P_{+}^{I}$, an equilibrium is an $(I+1)$-tuple $\left(z_{1}, \ldots, z_{I}, q\right)$ satisfying (3.19) and (3.20). Let $N$ denote the set of points in $P_{+}^{I}$ for which there exists a corresponding equilibrium such that for some trader $i$ and asset $k$

$$
z_{i}^{k}=0 \quad \text { and } \quad V_{i k}\left(z_{i}\right)=0 .
$$

To prove the lemma, it is sufficient to show that $N$ is negligible in $P_{+}^{I}$. Actually, I shall show that $N$ is contained in a larger set, $N^{*}$, which is also negligible. To define $N^{*}$, for every trader $i$ let $\mathscr{K}_{i}$ by any (possibly empty) subset of the commodities $1, \ldots, K$, and consider the following equations:

$$
\begin{aligned}
& V_{i k}\left(z_{i}\right)=0, \text { for all } k \text { in } \mathscr{K}_{i}, \\
& z_{i}^{k}=0, \text { for all } k \text { not in } \mathscr{K}_{i} .
\end{aligned}
$$

If $\left(\pi_{i}\right)$ is in $N$, then there is an associated equilibrium, and sets $\mathscr{K}_{1}, \ldots, \mathscr{K}_{I}$, such that (3.20) is satisfied, (3.22) is satisfied for all $i$, and in addition there is some trader $j$ and some commodity $n$ in $\mathscr{K}_{j}$ such that $z_{j}^{n}=0$. This motivates the 
following definition; in this definition I show explicitly that $V_{i k}\left(z_{i}\right)$ depends also on $q$ and $\pi_{i}$. For any sets $\mathscr{K}_{1}, \ldots, \mathscr{K}_{I}$, any one trader $j$, and any commodity $n$ in $\mathscr{K}_{j}$, let $N^{*}\left(\mathscr{K}_{1}, \ldots, \mathscr{K}_{1}, j, n\right)$ be the set of points $\left(\pi_{i}\right)$ in $P_{+}^{I}$ such that the following equations have a solution in $z_{i}, \ldots, z_{I}, q$ :

$$
\left.\begin{array}{l}
\sum_{i}\left(z_{i}-w_{k}\right)=0, \\
V_{i k}\left(z_{i}, q, \pi_{i}\right)=0, \quad k \text { in } \mathscr{K}_{i} \\
z_{i}^{k}=0, \quad k \operatorname{not} \text { in } \mathscr{K}_{i} \\
z_{j}^{n}=0 .
\end{array}\right\} \text { all } i,
$$

Further, define $N^{*}$ to be the union of all the (finitely many) sets $N^{*}\left(\mathscr{K}_{1}, \ldots, \mathscr{K}_{I}, j, n\right)$; then $N$ is a subset of $N^{*}$. To show that $N^{*}$ is negligible, it suffices to show that each $N^{*}\left(\mathscr{K}_{1}, \ldots, \mathscr{K}_{I}, j, n\right)$ is negligible.

To that end, fix $\mathscr{K}_{1}, \ldots, \mathscr{K}_{I}, j$, and $n$ (with $n$ in $\mathscr{K}_{j}$ ), and let $G$ be the mapping from $L^{*} \equiv R^{(I+1) K} \times P_{+}^{I}$ to $R^{(I+1) K+1}$ whose value at a point $\left(z_{1}, \ldots, z_{I}, q, \pi_{1}, \ldots, \pi_{I}\right)$ in $L^{*}$ is given by the left side of (3.23). Let $M^{*}$ be the set of points in $L^{*}$ at which $G$ is zero. $G$ is clearly continuously differentiable. I shall show that the Jacobian of $G$ has rank $(I+1) K+1$ at every point of $M^{*}$. With this fact, and an argument just like that used to prove the Proposition, it follows that $M^{*}$ is a differentiable manifold of dimension equal to $\operatorname{dim}\left(\Pi^{I}\right)-1$, and hence that the coordinate projection of $M^{*}$ in $P_{+}^{I}$ is negligible.

To show that $J(G)$, the Jacobian of $G$, has full rank, I start by displaying a block decomposition of $J(G)$ in the following table, where the rows correspond to the lines of (3.23), and the columns to $z_{1}, \ldots, z_{I}, q, \pi_{1}, \ldots, \pi_{I}$. The symbol 1 denotes the $K \times K$ identity matrix, and the symbol $n$ denotes the $n^{\prime}$ th unit vector (column) in $R^{K}$. For simplicity, and without loss of generality, I take $j=1$.

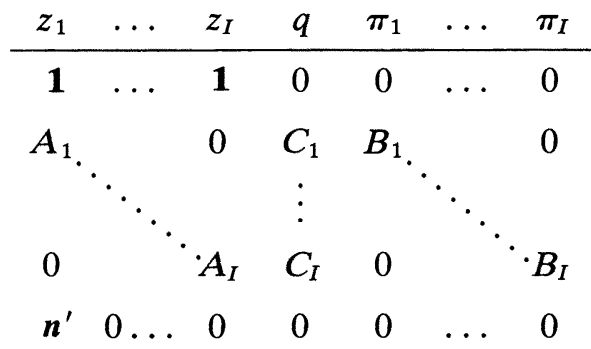

I want to show that the set of all linear combinations of the columns of $J(G)$ that can be obtained by admissible variations in the vectors $z_{i}, q$, and $\pi_{i} \operatorname{span} R^{(I+1) K+1}$. Note that, by the definition of the mapping $G$, the vectors $z_{i}$ and $q$ vary in $R^{K}$, but each vector $\pi_{i}$ varies in $P_{+}$. Hence, any "differential" $d \pi_{i}$ of $\pi_{i}$ must satisfy

$$
\sum_{e} d \pi_{i e}=0 .
$$


Consider first a matrix $B_{i}$; its elements are given by

$$
B_{i}^{h e}=\left\{\begin{array}{l}
\frac{\partial V_{i h}}{\partial \pi_{i e}}=U_{i}^{\prime}\left(v_{e}^{\prime} z_{i}\right) v_{e}^{h}, \quad h \in \mathscr{K}_{i}, \\
0, \quad h \notin \mathscr{K}_{i} .
\end{array}\right.
$$

Define $X$ to be the set of all vectors $x=B_{i} \beta$ such that $\beta=\left(\beta_{e}\right)$ satisfies $\Sigma_{e} \beta_{e}=0 . X$ clearly is contained in $K_{i}$, the coordinate subspace of $R^{K}$ corresponding to the commodities in $\mathscr{K}_{i}$. I shall now show that $X$ spans $K_{i}$. If not, there would be a vector $\bar{x} \neq 0$ in $K_{i}$ orthogonal to $X$; in other words $\Sigma_{e} \beta_{e}=0$ would imply $\bar{x}^{\prime} B_{i} \beta=0$. This would imply that all of the coordinates of $\bar{x}^{\prime} B_{i}$ would be equal, i.e. that all of the numbers $\bar{x}^{\prime} v_{e} U_{i}^{\prime}\left(v_{e}^{\prime} z_{i}\right)$ would be equal; but this would contradict part (b) of Assumption (A3). (Note that $\bar{x}^{\prime} v_{e} U_{i}^{\prime}\left(v_{e}^{\prime} z_{1}\right)>0$, since $\bar{x} \neq 0$ and $U_{i}^{\prime}\left(v_{e}^{\prime} z_{i}\right) v_{e} \gg 0$.) Hence, $X$ spans $K_{i}$.

Let $\bar{B}_{i}$ denote the matrix of columns of $J(G)$ corresponding to $\pi_{i}$, and let $B$ be the subspace of $R^{(I+1) K+1}$ spanned by all vectors of the form $\Sigma_{i} \bar{B}_{i} \beta_{i}$ such that, for each $i, \Sigma_{e} \beta_{i e}=0$. From what has just been shown in the preceding paragraph, $B$ is a coordinate subspace of $R^{(I+1) K+1}$, isomorphic to the product of the $K_{i}$, and of dimension $\Sigma_{i}\left(\# \mathscr{K}_{i}\right)$. It remains to find additional columns of $J(G)$ that, together with $B$, span $R^{(I+1) K+1}$. This can be done by selecting a set of columns of $J(G)$ corresponding to $z_{1}, \ldots, z_{I}$. I omit the details.

Proof of Lemma 2: The technique is similar to that used in Lemma 1. Recall that the mapping $F$ is defined by (3.14). The notation will be slightly different from that used in Lemma 1 . The vectors $q_{s}(s=1,2)$ denote the price vectors corresponding to the two signals, and $\pi_{s}$ is the array $\left(\pi_{i s e}\right)$ of probabilities, with $\Sigma_{e} \pi_{i s e}=1$, all $s, i$. A block decomposition of the Jacobian $J(F)$ is shown in the following table, where the rows correspond to the coordinates of the value of $F$, and the columns to $q_{1}, q_{2}, \pi_{1}$, and $\pi_{2}$. Again, the symbol 1 denotes the $K \times K$ identity matrix.

\begin{tabular}{cccc}
$q_{1}$ & $q_{2}$ & $\pi_{1}$ & $\pi_{2}$ \\
\hline$A_{1}$ & 0 & $B_{1}$ & 0 \\
0 & $A_{2}$ & 0 & $B_{2}$ \\
$\mathbf{1}$ & $-\mathbf{1}$ & 0 & 0
\end{tabular}

(The symbols $A_{s}$ and $B_{s}$ will have different meanings than in Lemma 1.) I want to show that the set of all linear combinations of the columns of (3.27) that can be obtained by admissible variations in $q_{1}, q_{2}, \pi_{1}$, and $\pi_{2}$ span $R^{3 K}$. Recall that $q_{1}$ and $q_{2}$ are each in $R_{++}^{K}$, and $\pi_{1}$ and $\pi_{2}$ are each in $\mathscr{D}$. Any "differential" $d \pi_{s}$ of $\pi_{s}$ must satisfy

$$
\sum_{e} d \pi_{i s e}=0, \quad \text { all } s, i .
$$

This motivates the following language: I shall say that a submatrix of the part of $J(F)$ corresponding to $\pi_{1}$ and $\pi_{2}$ has rank $r$ if the set of linear combinations of the 
columns of that submatrix whose coefficients satisfy (3.28) spans a subspace of dimension $r$.

First, the columns corresponding to $q_{1}$ are independent and have rank $K$, because of the identity block at the bottom, and are also independent of all columns corresponding to $\pi_{1}$ and $\pi_{2}$. Second, the columns corresponding to $\pi_{1}$ are independent of the columns corresponding to $\pi_{2}$. Hence it is sufficient to show that $B_{1}$ and $B_{2}$ each have rank $K$.

Each matrix $B_{s}$ is composed of $I$ groups of columns; each group $i$ forms a submatrix, the Jacobian of $i$ 's excess demand function with respect to $\pi_{i s}$, which is equal to the corresponding Jacobian of $i$ 's gross demand function, since the endowment of $i$ (given $s$ ) is fixed.

Consider the first-order conditions for a particular trader's optimal demand, $(c, z)$, given an asset price vector $q \gg 0$, and given the trader's probability vector $p \equiv\left(p_{e}\right) \equiv \pi_{i s} \gg 0$. To simplify the notation, I shall suppress the indices $i$ and $s$. Let $y_{e} \equiv v_{e}^{\prime} z$ (the value of the portfolio $z$ in state $e$ ). The first-order conditions for the optimal $(c, z)$ are given by (3.19), which I rewrite here as

$$
\begin{array}{lll}
z \geqslant 0, & & \\
z^{k}>0 & \text { implies } & V_{k}(z)=0, \\
z^{k}=0 & \text { implies } & V_{k}(z) \leqslant 0,
\end{array}
$$

where

$$
\begin{aligned}
& V(z) \equiv U_{0}(c)+\sum_{e} p_{e} U\left(y_{e}\right), \\
& c=w_{0}+q^{\prime} w-q^{\prime} z, \\
& y_{e}=v_{e}^{\prime} z,
\end{aligned}
$$

and $V_{k}$ is the partial derivative of $V$ with respect to $z^{k}$ (taking account of the dependence of $c$ and $y_{e}$ on $z$ ). These partial derivatives are given by

$$
V_{k}(z)=-U_{0}^{\prime}(c) q^{k}+\sum_{e} p_{e} U^{\prime}\left(y_{e}\right) v_{e}^{k}
$$

Since $p$ is in $\mathscr{D}$, it follows from the way $\mathscr{D}$ was constructed in Lemma 1 that the third line of (3.29) can be sharpened to read:

$$
z^{k}=0 \text { implies } V_{k}(z)<0 .
$$

As in the proof of Lemma 1, let $\mathscr{K}\left(=\mathscr{K}_{i}\right)$ be the set of assets such that $z^{k}>0$.

We shall think of $z, c$, and $y_{e}$ as functions of $p$. Let $f$ be a particular state of the environment; if we differentiate the first order conditions (3.29)-(3.32) with respect to $p_{f}$ we get

$$
\begin{aligned}
& \left(D V_{k}(z)\right)\left(\frac{\partial z}{\partial p_{f}}\right)+U^{\prime}\left(y_{f}\right) v_{f}^{k}=0, \quad k \in \mathscr{K}, \\
& \frac{\partial z^{k}}{\partial p_{f}}=0, \quad k \notin \mathscr{K} .
\end{aligned}
$$


One easily verifies that

$$
D^{2} V(z)=D^{2} \bar{U}(z)+U_{0}^{\prime \prime}(c) q q^{\prime} \equiv Q .
$$

It has already been noted, in (3.13), that $D^{2} \bar{U}(z)$ is negative definite. Therefore, since $U_{0}^{\prime \prime}(c) q q^{\prime}$ is negative semidefinite, $Q$ is negative definite, and hence nonsingular. Let $Q^{\mathscr{K}}$ be the square submatrix obtained from $Q$ by deleting the rows and columns corresponding to assets $k$ not in $\mathscr{K}$, and let $z^{\mathscr{K}}$ and $v_{f}^{\mathscr{K}}$ be similarly defined. Then one can rewrite (3.33) as

$$
\begin{aligned}
& Q^{\mathscr{K}}\left(\frac{\partial z^{\mathscr{K}}}{\partial p_{f}}\right)=-U^{\prime}\left(y_{f}\right) v_{f}^{\mathscr{K}}, \\
& \frac{\partial z^{k}}{\partial p_{f}}=0 \text { for } k \notin \mathscr{K} .
\end{aligned}
$$

Hence

$$
\begin{aligned}
& \left(\frac{\partial z^{\mathscr{K}}}{\partial p_{f}}\right)=-\left(Q^{\mathscr{K}}\right)^{-1} U^{\prime}\left(y_{f}\right) v_{f}^{\mathscr{K}}, \\
& \frac{\partial z^{k}}{\partial p_{f}}=0, \text { for } \quad k \notin \mathscr{K} .
\end{aligned}
$$

By the argument used in Lemma 1, the following equation (3.26), the set of vectors

$$
\left\{\sum_{f} \beta_{f} U^{\prime}\left(y_{f}\right) v_{f}^{\mathscr{K}}: \sum_{f} \beta_{f}=0\right\}
$$

is a linear space of dimension \# $\mathscr{K}$. Hence the Jacobian $(\partial z / \partial p)$ spans the coordinate subspace of $R^{K}$ corresponding to assets in $\mathscr{K}$. For trader $i$, call this subspace $\mathscr{H}_{i}$.

One then repeats this argument for another trader whose equilibrium portfolio includes an asset not demanded by trader $i$. One continues in this way until all assets have been accounted for, which must eventually happen because the total demand for every asset is positive. Thus the subspaces $\mathscr{H}_{i}$ will span $R^{K}$.

\section{COMMENTS ON THE ASSUMPTIONS}

The basic proposition of this paper is that generically, i.e., except for a negligible set of economies that satisfy the assumptions of the model, there exists a rational expectations equilibrium whose prices are "revealing," i.e. reveal to all market agents all the information initially available to all the agents. My comments on the assumptions address two issues: (i) Does Assumption 3 characterize a set of economies whose complement is negligible in the broader set of economies that otherwise fit the formulation of the model and satisfy Assumptions 1 and 2? (ii) How important is the requirement of the model that the sets of information signals be finite? 
To begin the discussion of the first issue, let me recall that there are really two sets of assumptions. One set is implicit in the formulation of the model, the other is described in Assumptions 1-3. Given the formulation of the model, the following objects comprise the data of a particular economy:

(1) (a) The finite number $I$ of traders, and the finite number $K$ of assets; (b) the traders' utility functions, $U_{0 i}$ and $U_{i}$, and their endowments, $\omega_{i}$.

(2) The finite number, say $H$, of alternative payoff-relevant environments, and the finite numbers of alternative information signals in the several traders information sets, say $J_{1}, \ldots, J_{I}$.

(3) The array $\left(v_{e}\right)$ of vectors of eventual asset values in the several environments.

(4) The array $\pi$ of conditional probabilities $\pi_{\text {ise }}$.

Thus, within the model, an economy is characterized by an array

$$
\left[I, K,\left(U_{0 i}, U_{i}, \omega_{i}\right), H,\left(J_{i}\right),\left(v_{e}\right),\left(\pi_{i s e}\right)\right] .
$$

Let $A$ denote the set of economies.

If one fixes the number of traders, their utility functions and endowments, and the numbers of environments and signals, then one obtains a smaller set of economies, say $A^{\prime}$. (The set $A^{\prime}$ depends, of course, on the just-mentioned data, and should really be written as

$$
\left.A^{\prime}\left[I, K,\left(U_{0 i}, U_{i}, \omega_{i}\right), H,\left(J_{i}\right)\right] .\right)
$$

Within any fixed set $A^{\prime}$, an economy is characterized by the array $\left[\left(v_{e}\right),\left(\pi_{i s e}\right)\right]$. Let $V$ denote the set of all nonnegative arrays $\left(v_{e}\right)$, given $K$ and $H$, and let $\Pi$ denote the set of all nonnegative arrays $\left(\pi_{\text {ise }}\right)$ such that

$$
\sum_{e} \pi_{i s e}=1, \quad \text { for all } i \text { and } s .
$$

Then $V$ has dimension $K \times H, \Pi$ has dimension $I(H-1) J_{1} \times \ldots \times J_{1}$, and an economy in (a fixed set) $A^{\prime}$ is characterized by a point in $V \times \Pi$.

Let $A^{\prime}$ be fixed with $H>K$. The first thing to note is that the set of arrays $\left(v_{e}\right)$ satisfying Assumption A3(a) is open and of full measure in $V$. Hence, if $H>K$, the set of economies not satisfying Assumption A3(a) is negligible in $A^{\prime}$.

Turning to Assumption A3(b), one would hope that, with additional regularity conditions on the utility functions, this assumption would be satisfied for all but a negligible set of economies in any given set $A^{\prime}$ (provided $H>K$ and Assumptions $\mathrm{A} 1$ and $\mathrm{A} 2$ are also satisfied). However, I have no result of this kind at present.

To conclude the discussion of the first issue, let me suggest that for some purposes the model has allowed the set $\Pi$ of arrays $\left(\pi_{\text {ise }}\right)$ to be too large. Suppose that all traders agree on the conditional probabilities of (joint) signals $s$ given environments $e$, but have possibly different "prior" probabilities for the environments. In this case the dimension of $\Pi$ would be smaller, and the demonstration that a set is negligible in $\Pi$ would be a sharper result. 
I turn now to the second issue: is it important that the sets of information signals be finite? The first thing to note is that one cannot expect in general that equilibrium prices will be revealing if the signal sets are "too large." For example, if the sets of signals were Euclidean spaces, and the equilibrium prices were smooth functions of the joint signal, then the equilibrium prices could not be revealing if the dimension of the joint signal exceeded the number of commodities.

Whether or not rational expectations equilibria exist generically, in some fairly general model, is an open question. If the sets of signals are not finite, then $\Pi$ is infinite dimensional. In this case, the concept of "negligible" set in $\Pi$ is not straightforward; there will typically be a choice of natural topologies on $\Pi$, and there may be no natural measure corresponding to Lebesgue measure. J. Green [4] has given an example with infinite signal spaces in which no rational expectations equilibrium exists, and stimulated by Green's example Jordan and Radner [9] have constructed an example with infinite $E$ and $S$ in which the set of economies (suitably topologized) for which no rational expectations equilibrium exists has a nonempty interior.

\section{Harvard University}

Manuscript received August, 1977; revision received March, 1978.

\section{REFERENCES}

[1] BEJA, A.: "The Limited Information Efficiency of Market Processes," Working Paper No. 43, Research Program in Finance, University of California, Berkeley, May, 1976.

[2] Chichilnisky, G., and P. J. Kalman: "Properties of Critical Points and Operators in Economics," Journal of Mathematical Analysis and Applications, 57 (1977), 340-349.

[3] GREEN, J.: "Information, Efficiency and Equilibrium," Harvard Institute of Economic Research, Discussion Paper 284, March, 1973.

[4] - "The Non-Existence of Informational Equilibria," Review of Economic Studies, 44 (1977), 451-463.

[5] Grossman, S.: "The Existence of Futures Markets, Noisy Rational Expectations and Informational Externalities," Ph.D. dissertation, University of Chicago, Department of Economics, 1975.

[6] Grossman, S., AND J. E. Stiglitz: "Information and Competitive Price Systems," American Economic Review, 66 (1976), 246-253.

[7] JoRDAN, J.: "On the Predictability of Economic Events," University of Pennsylvania, Department of Economics, Discussion Paper 329, February, 1976 (to appear in Econometrica).

[8] ——: "Expectations Equilibrium and Informational Efficiency for Stochastic Environments," Journal of Economic Theory, 16 (1977), 354-372.

[9] JORDAN, J., AND R. RADNER: "Example of Nonexistence of Rational Expectations Equilibrium," Institute for Mathematical Studies in the Social Sciences, Stanford University, July 21, 1977 (unpublished notes).

$[\because \rightarrow$ KinlSTROM, R. E., AND L. J. MiRMAN: "Information and Market Equilibrium," Bell Journal of Economics, 6 (1975), 357-376.

[11] KREPS, D.: "A Note on 'Fulfilled Expectations' Equilibria," Journal of Economic Theory, 14 (1977), 32-43.

[12] MARschAK, J.: "The Payoff-Relevant Description of States and Acts," Econometrica, 31 (1963), 719-725. 
[13] RADNER, R.: "Equilibre des Marchés à Terme et au Comptant en Cas d'Incertitude," Cahiers d'Econometrie, 1967, C.N.R.S., Paris 35-52 (translated as "Equilibrium of Spot and Futures Markets under Uncertainty," Center for Research in Management Sciences, University of California, Berkeley, Technical Report 24, April, 1967).

[14] — : "Rational Expectations Equilibrium with Imperfect Market Models" (in preparation), 1977; preliminary version presented at Bell Laboratories, Holmdel, N.J., September, 1976.

[15] SHILleR, R.: "Rational Expectations and the Dynamic Structure of Macroeconomic Models: A Critical Review," Journal of Monetary Economics, 4 (1978), 1-44.

[16] STERnBerG, S.: "Differentiable Manifolds," Chapter II in Lectures on Differentiable Geometry. Englewood Cliffs, N.J.: Prentice-Hall, Inc., 1964, pp. 31-96. 